\title{
Integration of atom interferometers and inertial measurement units to improve navigation performance
}

\author{
$1^{\text {st }}$ Benjamin Tennstedt \\ Institut für Erdmessung \\ Leibniz Universität Hannover \\ Hanover, Germany \\ tennstedt@ife.uni-hannover.de
}

\author{
$2^{\text {nd }}$ Steffen Schön \\ Institut für Erdmessung \\ Leibniz Universität Hannover \\ Hanover, Germany \\ schoen@ife.uni-hannover.de
}

\begin{abstract}
This paper explores a way of combining conventional inertial sensors with cold atom interferometers (CAI) in order to reduce the drift of the navigation solutions in velocity and orientation. Instead of complementing and improving the CAI with conventional sensors, in this approach the conventional IMU will be used as main sensor for a prediction of the kinematic state. The CAI is then used for the correction of systematic errors and offsets in the framework of an extended Kalman Filter. Monte Carlo simulation studies demonstrate an improvement of the navigation solution precision. In addition, most drifts of velocity and orientation can be eliminated and the uncertainty of the velocity solution can further be reduced by a factor of 30 or more compared to the conventional strapdown. The observability of the error states is discussed.
\end{abstract}

Index Terms-Inertial navigation, Cold atom interferometry, Hybridization, Extended Kalman Filter

\section{INTRODUCTION}

Inertial measurement units (IMU) are an excellent choice for many navigation tasks due to their short term accuracy and autonomous measurement principle. However, the signal processing step of integrating base signals, i.e. accelerations and turn rates, leads to a large drift of the navigation solution, namely position, velocity and attitude.

One way to reduce this drift is the combination with other absolute sensors like global navigation satellite systems (GNSS), which on the other hand leads to a loss of independence from external systems and does not work in cases without direct line-of-sight to the satellites.

One promising option are atom interferometers [1]. As inertial sensors they provide superior long-term stability and high sensitivity [2], and are already established as one-axis acceleration sensors, i.e. quantum gravimeters [3], or even commercial [4]. However, their use for high-rate navigation cases is limited due to the low datarate caused by atom preparation times. Their low dynamic range makes them vulnerable to perturbations like mechanical vibrations leading to a potential loss of the target fringe interval.

Several attempts of a hybridisation have been made to overcome those effects [5], [6], mainly with the goal to use

This work was sponsored by BMWi, Project 50RK1957. the atom interferometer as main sensor, and support it with different conventional sensors in order to suppress noise and achieve maximum sensitivity and long-term stability in the case of vibrations and other disturbances of the signal.

However, another disadvantage of atom interferometry is the averaging effect of the measuring principle of summing the inertial effects on the wave packets travelling in the interferometry chamber. While this reduces the white noise density of the signal measurement in static cases with a constant acceleration or turn rate, in case of changing inertial quantities it leads to systematic errors due to sculling and coning effects [7]. An option to reduce the effects from the side of the atom interferometer is an increased datarate [8].

In the presented approach, the correction of systematic errors of the conventional IMU is focused on in order to improve the overall performance of the navigation solution. By means of a two-level strapdown algorithm [9], [10] with the acceleration and turn rate measurement of the conventional IMU, the target phase shift interval of the atom interferometer is predicted in order to solve the ambiguity issue of the interference fringes. This problem occurs if the dynamics of the trajectory are larger than the maximum forces that the atom interferometer can resolve within one fringe.

In a second step, systematic errors between the two sensors frames, like e.g., misalignments, leverarms and bias can be corrected by using the raw atom number output of the atom interferometer in an error state extended Kalman filter framework [7]. As a result, the actual fusion of the data of both sensors is accomplished in the observation space of the atom interferometer.

The remainder of the paper is structures as follows: the measurement principle of the atom interferometer is summarized in section II. Also, the state model of the atom wavepacket is highlighted, which enables a more precise calculation of the phase shift and thus reduces the systematic errors of the CAI. The hybrid filter is then summarized in section III. By means of Monte Carlo simulation studies, the performance gain of the novel filter approach for two different simulated trajectories is then demonstrated in III-B. The observability 


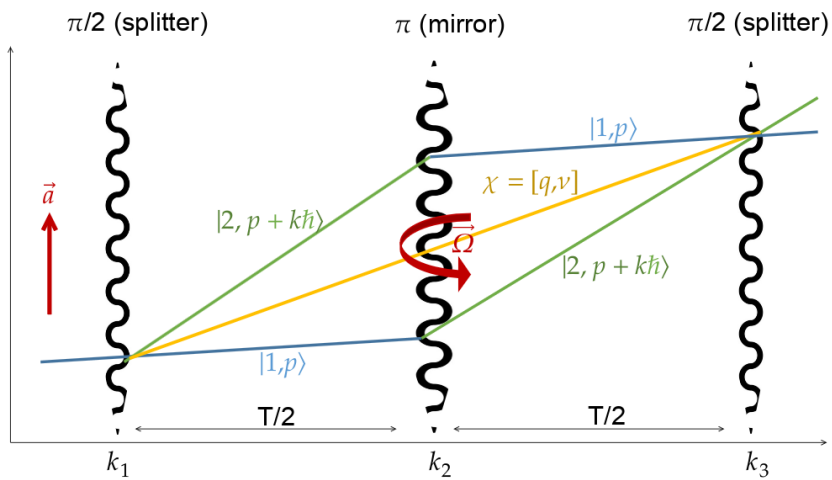

Fig. 1: Working principle of an atom interferometer in MachZehnder structure. After preparation, an atom wave packet is split into two states, reversed, and then recombined by directed Raman laser beams. The resulting phase shift at the third pulse yields information about any inertial force that affected the wave packets during their free evolution time. The two different atom states 1 (blue) and 2 (green), are illustrated in bra-ket notation. However, in the scope of this paper only the mean kinematic state $\chi$ (yellow) is needed.

requirements of a number of additional IMU error states are discussed in section IV. The results are finally summarized in section V.

\section{CAi Mathematical Model}

In literature (e.g. [11], [12]) the atom phase shift caused by inertial forces or turn rates is often expressed directly in relation to the inertial acceleration or turn rate. This mostly makes sense in scenarios with a nearly constant value like in gravimetry measurements.

However, there is a number of advantages to start with a more general equation where no assumption of a constant acceleration is necessary.

$$
\Phi=\mathbf{k} \cdot \mathbf{x}
$$

with - used to indicate element-wise multiplication. This equation describes the phase shift at an interaction of the Raman lasers (cf. fig. 1) with the atom packet at their position $\mathbf{x}$ in the light field. The effective laser wave vector in the corresponding direction, e.g. $k_{x} \sim 1 / \lambda$, is clearly defined by the laser system wavelength $\lambda$ in use.

In order to describe the motion of the atoms it is sufficient to use the mid-point line [13], here described by the state $\chi$ (cf. fig. 1).

For the atom motion state $\chi=[\mathbf{q}, \boldsymbol{\nu}]$ (with position $\mathbf{q}$ and velocity $\boldsymbol{\nu}$ relative to the body frame start position) only the integration of inertial effects in the b-frame is of interest. The differential equation system describing the temporal evolution of the state yields:

$$
\begin{aligned}
\dot{\mathbf{q}}^{b^{\prime}} & =\boldsymbol{\nu}^{b^{\prime}} \\
\dot{\boldsymbol{\nu}}^{b^{\prime}} & =\mathbf{C}_{b}^{b^{\prime}} \mathbf{f}_{i b}^{b}-\mathbf{C}_{b}^{b^{\prime}}\left(2 \boldsymbol{\omega}_{i b}^{b} \times \boldsymbol{\nu}^{b}\right) \\
\dot{\mathbf{C}}_{b}^{b^{\prime}} & =\mathbf{C}_{b}^{b^{\prime}}\left[\boldsymbol{\omega}_{i b}^{b} \times\right]
\end{aligned}
$$

The direction cosine matrix $C_{b}^{b^{\prime}}$ resembles the infinitesimal orientation change of the $b$-frame to its representation after the change $b^{\prime}$. The system of differential equations can be solved by trapezoid integration with the datarate of the conventional IMU as integration step width.

In the remainder of the paper, this calculation is labeled atom strapdown, since it resembles the same basic integration steps as a conventional strapdown algorithm [14].

The phase shift between the two atom paths is defined by each of the interactions of the atom clouds with the interrogation lasers [15]. The Mach-Zehnder configuration, cf. fig. 1 , uses a series of three laser pulses $\left(\frac{\pi}{2} ; \pi ; \frac{\pi}{2}\right)$ to split, reverse and recombine the two atom paths, i.e. their internal states. The final phase shift at the third and last atom-laser interaction yields:

$$
\Delta \boldsymbol{\Phi}\left(t_{3}\right)=\mathbf{k}_{1} \cdot \mathbf{q}\left(t_{1}\right)-2 \mathbf{k}_{2} \cdot \mathbf{q}\left(t_{2}\right)+\mathbf{k}_{3} \cdot \mathbf{q}\left(t_{3}\right)
$$

This also means that in case of symmetric Raman pulses only the two atom positions at the atom-laser interactions need to be stored, since they influence the resulting phase shift: The one at $t=T / 2$, as well as the one at $t=T$. With those, and defining $\mathbf{k}_{1}=\mathbf{k}_{2}=\mathbf{k}_{3}=\mathbf{k}$, the total phase shift of the interferometer results to:

$$
\Delta \mathbf{\Phi}=-2 \mathbf{k} \cdot \mathbf{q}\left(\frac{1}{2} T\right)+\mathbf{k} \cdot \mathbf{q}(T)=\mathbf{k} \cdot\left(\mathbf{q}(T)-2 \mathbf{q}\left(\frac{1}{2} T\right)\right)
$$

In case of constant accelerations or turn rates, the position expressed by $\mathbf{q}=\frac{1}{2} \mathbf{a} t^{2}$ and $\mathbf{q}=\boldsymbol{\omega} \times \boldsymbol{\nu} t^{2}$ can be included in order to get the more familiar equation:

$$
\Delta \boldsymbol{\Phi}=\frac{1}{4} \mathbf{k} \cdot \mathbf{a} T^{2}+\frac{1}{2} \boldsymbol{\omega} \times \boldsymbol{\nu} \cdot \mathbf{k} T^{2}
$$

or expressed in terms of interrogation time $T_{\text {int }}=\frac{1}{2} T$ between the laser pulses:

$$
\Delta \mathbf{\Phi}=\mathbf{k} \cdot \mathbf{a} T_{i n t}^{2}+2 \boldsymbol{\omega} \times \boldsymbol{\nu} \cdot \mathbf{k} T_{i n t}^{2}
$$

with the first term based on linear accelerations and the second term resembling Coriolis effects due to rotations.

The actual output of the atom interferometer is not the phase directly, but a transition probability $p$ that is determined by counting the atoms in the excited state $N_{1}$ with respect to the total number of atoms $N_{1}+N_{2}$ [16].

$$
p=\frac{N_{1}}{N_{1}+N_{2}}
$$

It has the following relation to the interferometer phase shift:

$$
\mathbf{p}(\Delta \boldsymbol{\Phi})=A \cos \left(\Delta \boldsymbol{\Phi}+\boldsymbol{\Phi}_{0}\right)+\mathbf{p}_{0}
$$

This equation resembles the fringe pattern with $\boldsymbol{\Phi}_{0}$ as additional phase offset that can be varied with the interrogation laser control.

This already summarizes the physics package behind the atom interferometer. For the simulation experiments, two atom interferometers with their sensitive axis antiparallel w.r.t. each other are used to distinguish between linear acceleration and Coriolis forces [11], [12] ('Sagnac'-unit, cf. fig. 2). Another 


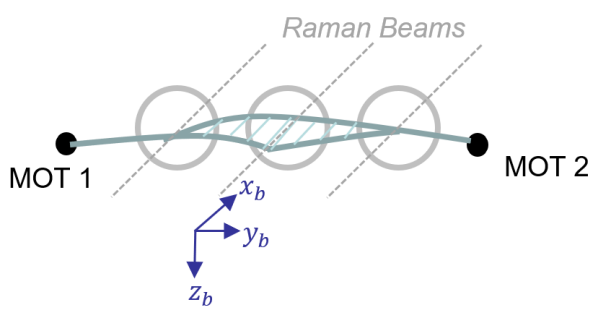

Fig. 2: Sagnac-unit of two counter-propagating interferometers. With this assembly it is possible to distinguish between linear acceleration and Coriolis effects. The atoms are prepared in the magneto-optical traps (MOT 1\&2), and propulsed towards each other.

option for differential interferometry with Bose-Einstein condensates is presented in [17].

Furthermore, three of those 'Sagnac'-units are set up perpendicular to each other in order to enable 6-degrees-offreedom (DOF) navigation capabilities, as well as full observability for the extended state vector as will be discussed in section IV. Another more sophisticated 6-DOF approach is presented in [18].

\section{HYBRID FILTER}

The general idea behind the hybridization is highlighted in several other works [5], [19]. The implementation in an error state navigation filter was explained in [7], the interested reader is referred to. In the following, a description about the general idea is given which is then formulated in a compact mathematical model. The signal flow chart in fig. 4 provides some intuition as well.

The studies in the next section will then add some further insight into the observation equations that also include the dynamical model used for the IMU measurements.

The general processing scheme is as follows:

1) The kinematic state is always propagated by a strapdown algorithm with the conventional IMU. This ensures that no information about the actual dynamics of the trajectory is lost in the cooldown and preparation times of the atom interferometer. As soon as the CAI measurement cycle starts, the algorithm switches to the extended Kalman filter in step 2;

2) First of all, the atom strapdown that was described before is used to get a prediction of the CAI phase shift as well as the atom population;

3) This predicted phase shift is then used as a steering signal for the laser phase $\phi_{L}$. This phase is set in a way that the resulting phase shift is exactly $\pi / 2$, cf. fig. 3. This makes sure that only one flank of the cosine is used in order to avoid another ambiguity. Also this mid-fringe region is the operation point with the highest sensitivity to changes and the least linearisation error;

4) The observation of the atom interferometer is then given by the transition probability $p$ as in eq. (10). With the already determined laser phase the measurement is shifted to the corresponding flank of the cosine in order to synchronize the predicted and the actual observation. Finally the actual filter step can be achieved in the CAI observation space.

The just described algorithm works as long as the difference of the CAI phaseshift and the predicted phase shift of the conventional IMU, including its errors like bias and random walk, is lower than $\frac{\pi}{2}$.

\section{A. Dynamic System}

The state vector reads:

$$
\mathbf{X}=\left[\mathbf{x}, \mathbf{v}, \Psi, \mathbf{b}_{a}, \mathbf{b}_{g}\right]^{T}
$$

with $\mathbf{x}$ and $\mathbf{v}$ as targeted 3-dimensional position and velocity expressed in the n-frame, respectively, $\Psi$ as total orientation with respect to the $\mathrm{n}$-frame and finally acceleration and gyro bias $\mathbf{b}_{a}, \mathbf{b}_{g}$, both defined in b-frame.

Sensor misalignments $\gamma$ and leverarms $\mathbf{l}$ are additional states that will be analysed in section IV, since they are not always observable and lead to some restrictions in the trajectory.

According to the general extended Kalman filter framework of [7], the nonlinear models of the strapdown as well as the observation equations are used to calculate the state vector.

For the propagation of the state uncertainties, the following transition matrix is used:

$$
\mathbf{\Phi}=\left(\begin{array}{ccccc}
\mathbf{I} & \mathbf{I} T & \mathbf{0} & \mathbf{0} & \mathbf{0} \\
& \mathbf{I} & \mathbf{0} & T \mathbf{C}_{b}^{n} & \mathbf{0} \\
& & \mathbf{I} & \mathbf{0} & T \mathbf{C}_{b}^{n} \\
& & & \mathbf{I} & \mathbf{0} \\
& & & & \mathbf{I}
\end{array}\right)
$$

and the observation matrix

$$
\mathbf{H}=\left(\begin{array}{cc}
-\frac{1}{2} A T \mathbf{k} \cdot \sin \left(\boldsymbol{\Phi}_{A}\right) & -A T \mathbf{k} \cdot \overline{\boldsymbol{\nu}} \cdot \sin \left(\boldsymbol{\Phi}_{A}\right) \\
-\frac{1}{2} A T \mathbf{k} \cdot \sin \left(\boldsymbol{\Phi}_{B}\right) & A T \mathbf{k} \cdot \overline{\boldsymbol{\nu}} \cdot \sin \left(\boldsymbol{\Phi}_{B}\right)
\end{array}\right)
$$

which will be further explained in section IV. Each entry of both matrices represents a $3 x 3$ matrix.

\section{B. Performance Simulation}

In order to evaluate the performance gain of the hybrid filter, two scenarios with different conditions were prepared. The first one is a typical train scenario with a nearly constant velocity of $40 \frac{\mathrm{m}}{\mathrm{s}}$ and just small changes of the orientation (cf. fig. 5). The second one is a slalom trajectory with a constant velocity of $1 \frac{\mathrm{m}}{\mathrm{s}}$ and a sinusoidal signal of the yaw rate as depicted in fig. 6 .

The simulation was performed for 100 Monte Carlo instances in parallel. Uncertainties according to [20] were added to the conventional IMU signal, with an additional bias for both acceleration and turn rates. To the observation of the atom interferometer an error from a normal distribution with variance $\sigma_{p}^{2}=10^{-12}$ is added, which is in the order of some actual experimental data [21].

In the train scenario, it can be clearly seen that the drift of the velocity in fig. $5 \mathrm{e}$ is almost completely compensated. The empirical standard deviation in fig. $5 \mathrm{~d}$ is reduced by factor 


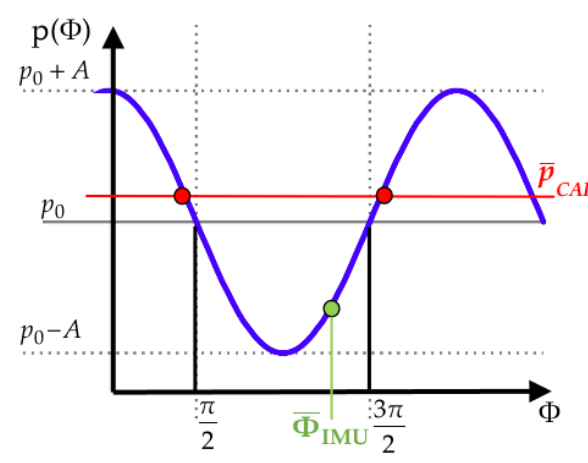

(a)

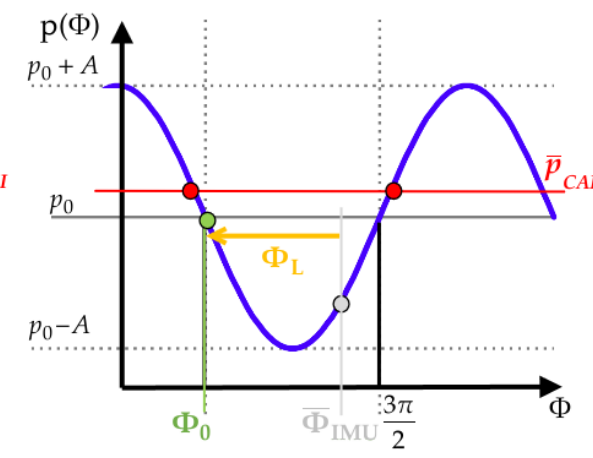

(b)

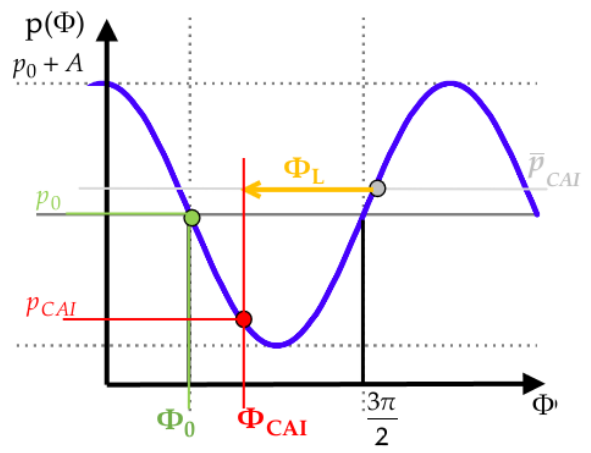

(c)

Fig. 3: Solution of the fringe ambiguity of the CAI measurement (red) at the filter observation update. Figure (a): First, the IMU data is used to calculate a predicted phase shift with the atom strapdown algorithm (green). Figure (b): The laser phase (yellow) is then set so that the predicted phase is exactly in the mid-fringe point at $\frac{\pi}{2}$. Figure (c): The value of this laser phase is then used to transfer the actual CAI observation to the same flank of the cosine signal, where the linearization of the filter equations is done.

30 as compared to the strapdown with the conventional IMU alone. The accelerometer bias (fig. 5b), as well as the bias of the gyroscope (fig. 5c), already reaches the reference value after a few iterations.

The systematic velocity drifts are more obvious in the slalom trajectory (fig. 6e).

The accelerometer bias (fig. 6b) again shows a quick convergence, as does the gyroscope bias (fig. 6c). However the changing dynamics lead to a larger uncertainty in the corner parts ( $\mathrm{min} / \mathrm{max}$ of the sinusoid trajectory) where the yaw rate reference signal reaches its peaks.

The overall performance gain of the velocity state (fig. 6d) still shows an improvement of factor 14 .

\section{ObSeRVABiLity AND StabiLity}

In the main filter, it is sufficient to only track the biases of the conventional IMU, since they already include any systematic offsets. However, one may be interested in the systematic displacement errors between the CAI unit and the conventional IMU.

The misalignment, scale error and the systematic offsets of the CAI unit alone can be estimated by conventional methods like the 6-position-test, if precise reference values for $\mathrm{g}$ and the local latitude are available.

This section will analyse the observability of the systematic errors misalignment and leverarms between the two sensor systems, as well as the bias of the classical sensors whose estimation was already empirically demonstrated in the simulations before.

The observation equations (14) of the CAI units are only coupled via $\delta \Phi$ with the state parameters.

$$
\mathbf{p}=\mathbf{A} \cdot \cos \left(\boldsymbol{\Phi}_{L}+\boldsymbol{\Phi}_{I M U}+\delta \boldsymbol{\Phi}\right)+\mathbf{p}_{0}
$$

We assume the following dynamic model to describe the velocity change:

$$
\dot{\mathbf{v}}=(\mathbf{I}-[\gamma \times])\left(\mathbf{f}^{\mathbf{b}}+\mathbf{b}_{\mathbf{a}}+\mathbf{f}_{\mathbf{r}}\right)
$$

where $f_{r}$ resemble fictitious forces due to a possible spatial displacement on the rigid body-frame (lever-arms), and the angular rate:

$$
\dot{\boldsymbol{\Psi}}=(\mathbf{I}-[\gamma \times])\left(\boldsymbol{\omega}^{\boldsymbol{b}}+\mathbf{b}_{\mathbf{g}}\right)
$$

The atom interferometer integrates all inertial influences during the flight time $T$. By reconsidering eq. 7 and assuming constant inertial forces, an average of the forces and turn rates is given by the increments $\Delta \mathbf{v}=\frac{\mathrm{a}}{T}$ and $\Delta \boldsymbol{\Psi}=\frac{\omega}{T}$.

$$
\Delta \mathbf{\Phi}=\frac{1}{2} \mathbf{k} \Delta \mathbf{v} T+\Delta \boldsymbol{\psi} \times \boldsymbol{\nu} \mathbf{k} T
$$

Some special care with the atom velocity $\nu$ is needed, since each interferometer axis needs their own 3D-velocity representation. As a result, the variable is replaced by $\bar{\nu}$, a vector filled with the atom velocities perpendicular to the respective sensitive axes. This then allows to replace the cross product in the second term with an element-wise multiplication with $\bar{\nu}$ which greatly simplifies the further notation.

The atom velocity also needs to be seen as constant in the scope of this analysis. This can be reasoned with a small calculation: The start velocity of the atoms is in the range of about $2.8 \mathrm{~m} / \mathrm{s}$ [16]. If an atom flight time of $0.02 \mathrm{~s}$ is chosen, even a large lateral acceleration of $1 \mathrm{~m} / \mathrm{s}^{2}$ would lead to a change of the velocity of under 1 percent which is neglectable.

With the adaptions described before, the phase shift can be expressed by:

$$
\Delta \boldsymbol{\Phi}=\frac{1}{2} \mathbf{k} \Delta \mathbf{v} T+\Delta \boldsymbol{\psi} \cdot \overline{\boldsymbol{\nu}} \cdot \mathbf{k} T
$$




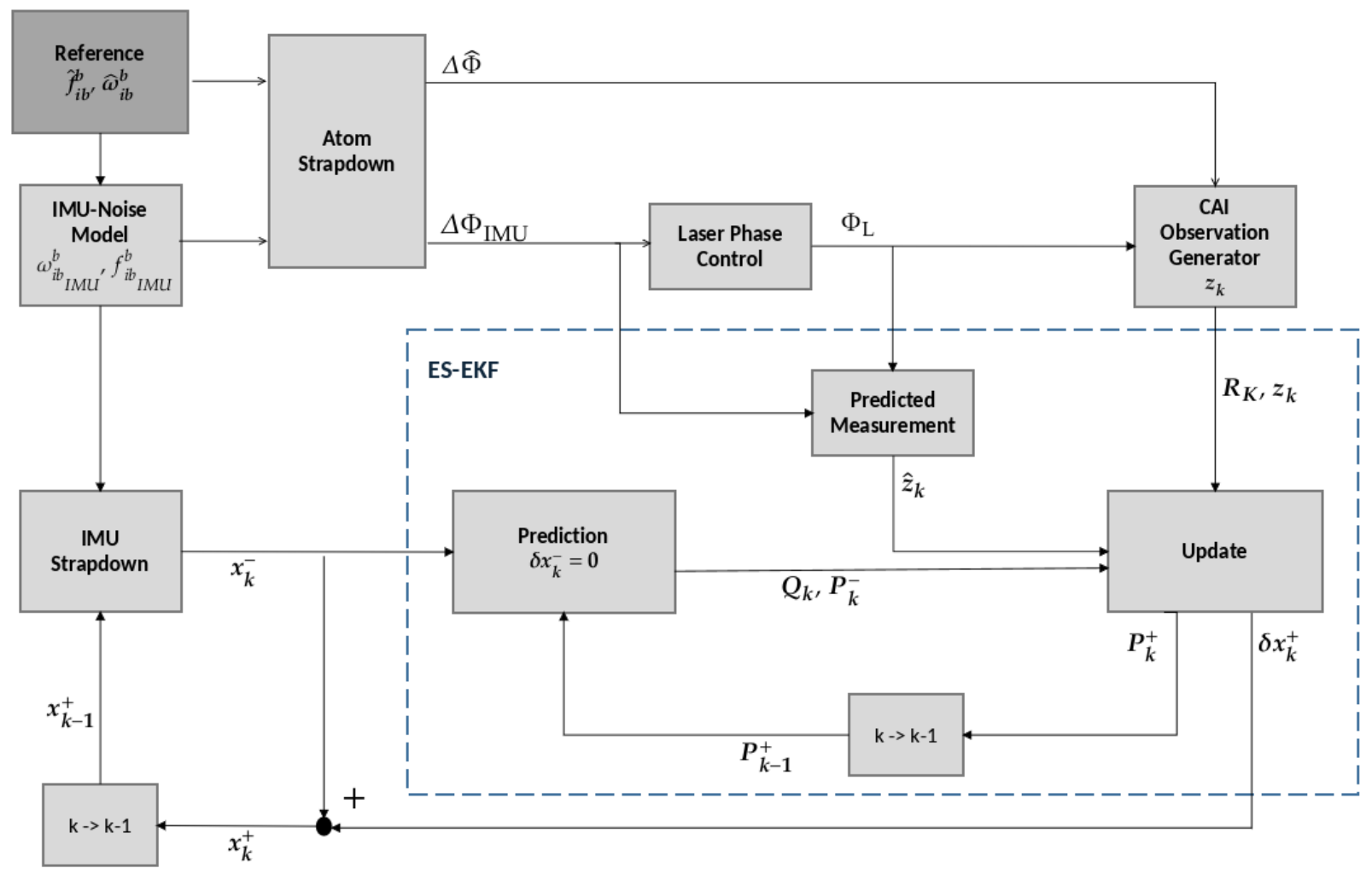

Fig. 4: Signal flow of the hybrid error state extended Kalman filter. The turn rates and specific forces of the reference trajectory are used to generate the CAI observation, based on the true phase shift determined by the atom strapdown algorithm, with noise added in the CAI observation generator. Simulated IMU data is generated by adding noise to the reference signals. This noisy data is then again used to predict the CAI phase shift according to the integrated IMU data. After setting the laser phase and synchronisation of the predicted and the actual measurement to the correct cosine fringe flank, the data is fused and the filter update generated. Finally, the update is added to the strapdown solution of the conventional IMU.

Back to the side of the IMU model. With constant inertial forces during $\mathrm{T}$, the equations (15) and (16) can be solved by simple integration in order to get:

$$
\begin{array}{r}
\delta \mathbf{v}=(\mathbf{I}-[\gamma \times]) \cdot\left(\mathbf{f}^{b}+\mathbf{b}_{a}+\mathbf{f}_{r}\right) \cdot T \\
\delta \mathbf{\Psi}=(\mathbf{I}-[\gamma \times]) \cdot\left(\boldsymbol{\omega}^{b}+\mathbf{b}_{g}\right) \cdot T
\end{array}
$$

If both quantities are set equal

$$
\begin{array}{r}
\delta \mathbf{v}=\Delta \mathbf{v} \\
\delta \boldsymbol{\Psi}=\Delta \boldsymbol{\Psi}
\end{array}
$$

the IMU error model can be included in the CAI observation equation. Then, the inertial phaseshifts as seen by the counterpropagating atom interferometers read:

$$
\begin{aligned}
\delta \boldsymbol{\Phi}_{A} & =\frac{1}{2} \mathbf{k} \cdot \delta \mathbf{v} T+\mathbf{k} \cdot \delta \boldsymbol{\Psi} \cdot \overline{\boldsymbol{\nu}} T \\
\delta \boldsymbol{\Phi}_{B} & =\frac{1}{2} \mathbf{k} \cdot \delta \mathbf{v} T-\mathbf{k} \cdot \delta \boldsymbol{\Psi} \cdot \overline{\boldsymbol{\nu}} T
\end{aligned}
$$

For the observability analysis of the IMU errors, the following state vector augmentation is defined:

$$
\mathbf{X}=\left[\mathbf{b}_{\mathbf{a}}, \mathbf{b}_{\mathbf{g}}, \boldsymbol{\gamma}, \mathbf{l}\right]^{T}
$$

Note that the kinematic states of position, velocity and orientation are not included here, since they are not observable with the atom interferometer in case of position, or just indirectly in case of the velocity and orientation via the error state [7].

Further, the observations of the CAI are given as

$$
\mathbf{Y}=\left[\mathbf{p}_{\mathbf{A}}, \mathbf{p}_{\mathbf{B}}\right]^{T}
$$

with $\mathbf{p}_{\mathbf{A}}=\left[p_{A x}, p_{A y}, p_{A z}\right]$ and $\mathbf{p}_{\mathbf{B}}$ analogous.

The partial derivatives $\partial \mathbf{Y} / \partial \mathbf{X}$ of the observation equations (10) w.r.t. the state vector (24) yield the observation matrix $\mathbf{H}$.

$$
\mathbf{H}^{T}=\left(\begin{array}{cc}
\frac{\partial}{\partial \mathbf{b}_{a}} A_{A} \cdot \cos \left(\Phi_{A}\right) & \frac{\partial}{\partial \mathbf{b}_{a}} A_{B} \cdot \cos \left(\Phi_{B}\right) \\
\frac{\partial}{\partial \mathbf{b}_{g}} A_{A} \cdot \cos \left(\Phi_{A}\right) & \frac{\partial}{\partial \mathbf{b}_{g}} A_{B} \cdot \cos \left(\Phi_{B}\right) \\
\frac{\partial}{\partial \gamma} A_{A} \cdot \cos \left(\Phi_{A}\right) & \frac{\partial}{\partial \gamma} A_{B} \cdot \cos \left(\Phi_{B}\right) \\
\frac{\partial}{\partial \mathbf{l}} A_{A} \cdot \cos \left(\Phi_{A}\right) & \frac{\partial}{\partial \mathbf{l}} A_{B} \cdot \cos \left(\Phi_{B}\right)
\end{array}\right)
$$

The observability of any state is given as long as the corresponding state can be reached by an observation in $\mathbf{Y}$ via the observation matrix $\mathbf{H}$.

In the scope of this analysis, no dynamic model for the propagation of the augmented states is defined, hence the transition matrix equals the unity and it is sufficient to take a look on $\mathbf{H}$ for further analysis. 


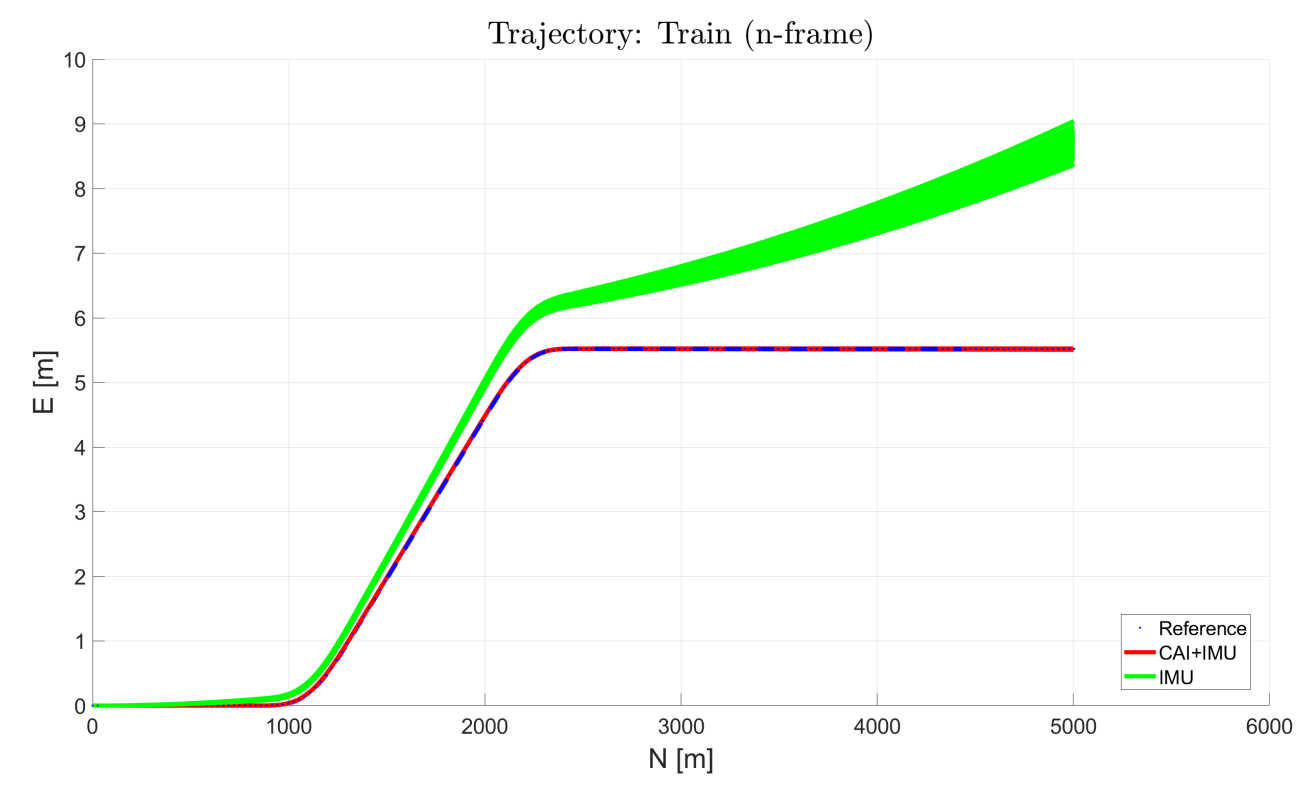

(a)

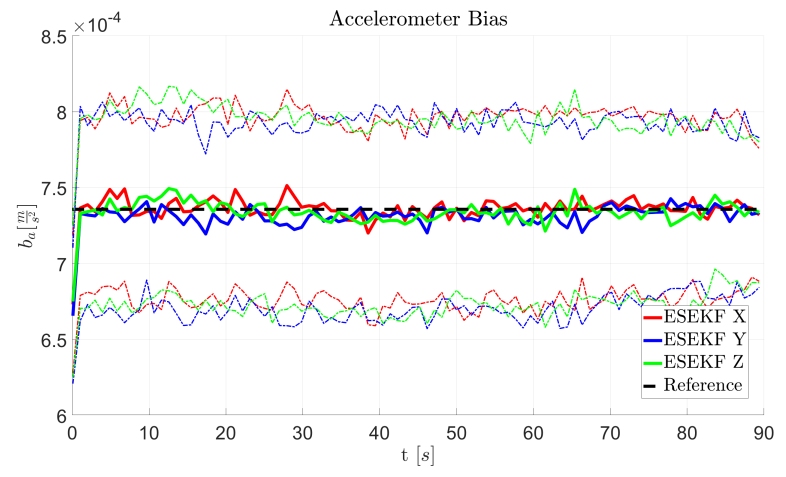

(b)

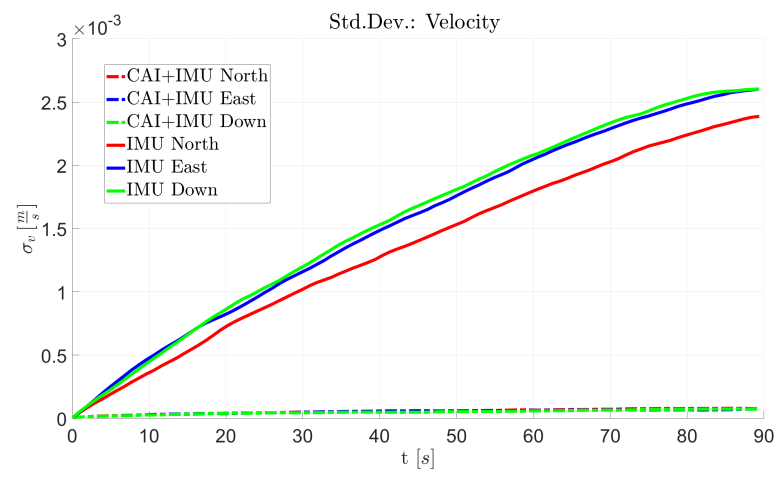

(d)

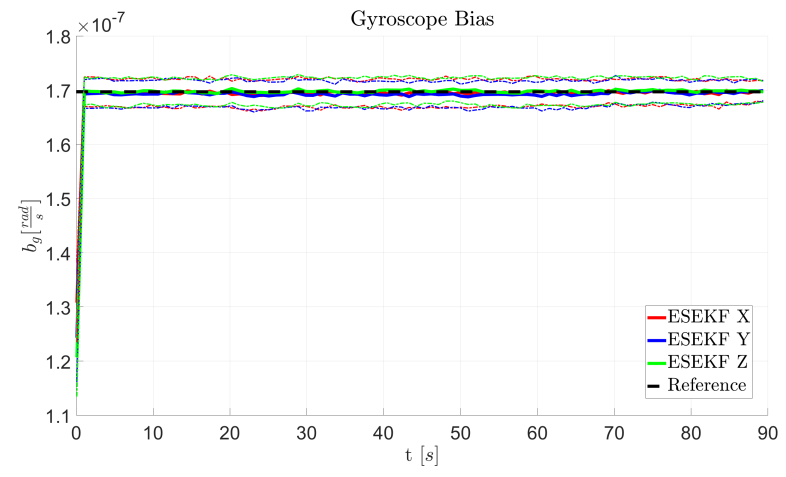

(c)

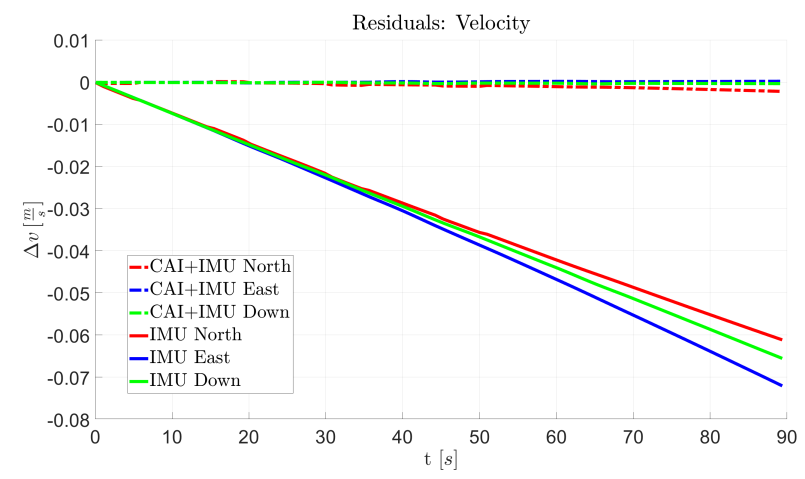

(e)

Fig. 5: Simulated trajectory of a train (a) with a velocity of $40 \mathrm{~m} / \mathrm{s}$ and minor changes in acceleration and orientation. The biases of accelereration (b) and turn rate (c) are converging nearly instantly, with the dotted lines indicating the empirical standard deviation. The uncertainty of the velocity state estimate is reduced by factor of 30 (d), and the systematic drift of the velocity strongly reduced (e). 


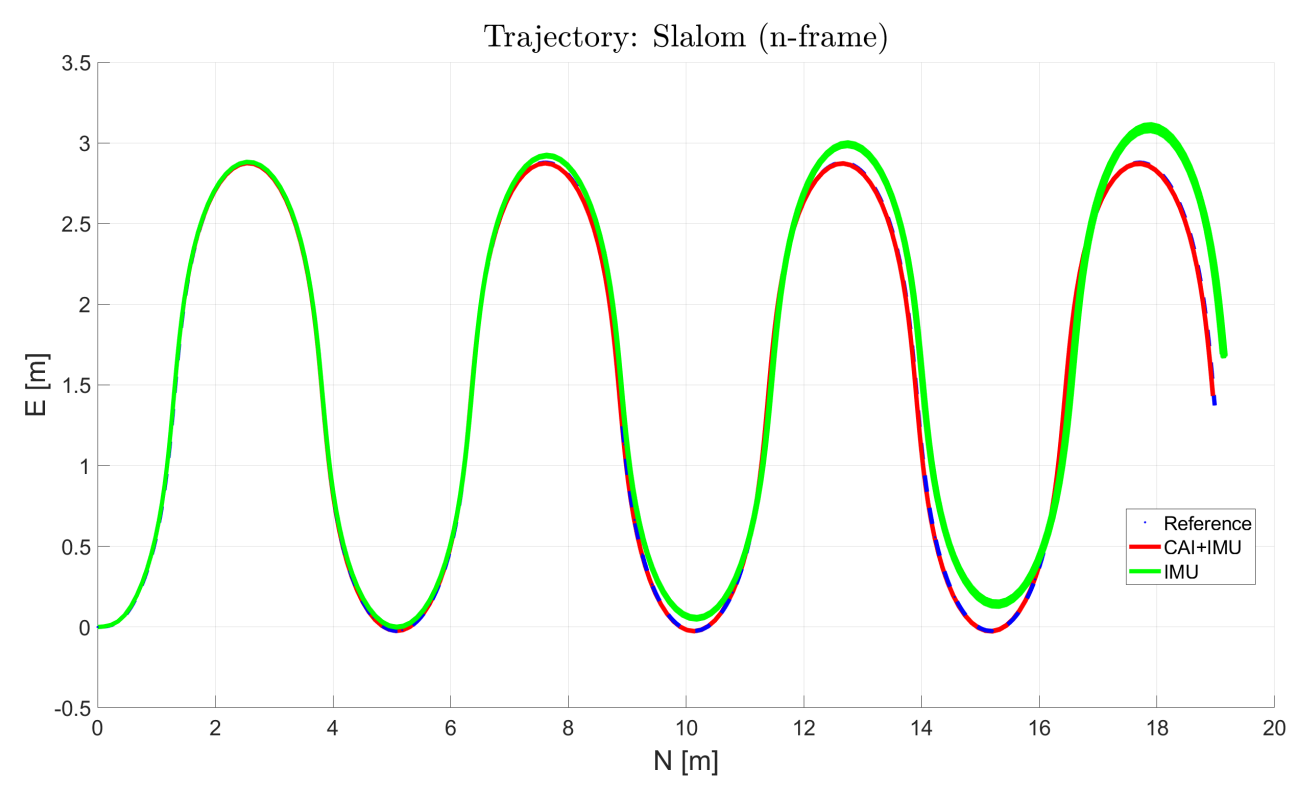

(a)

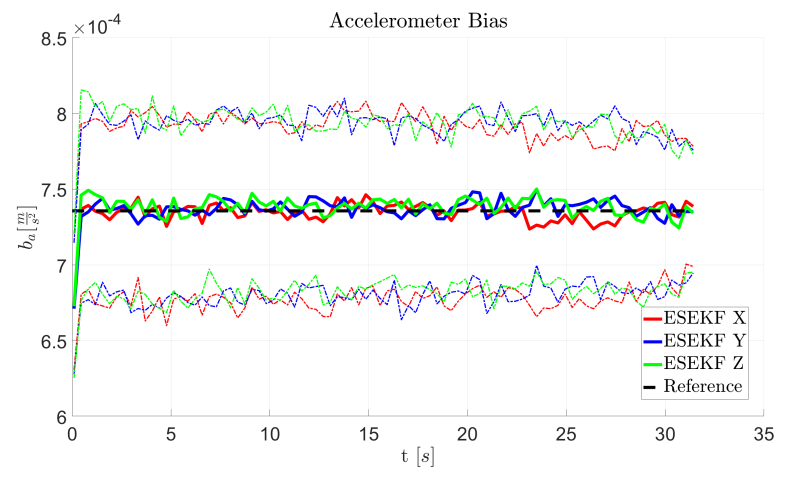

(b)

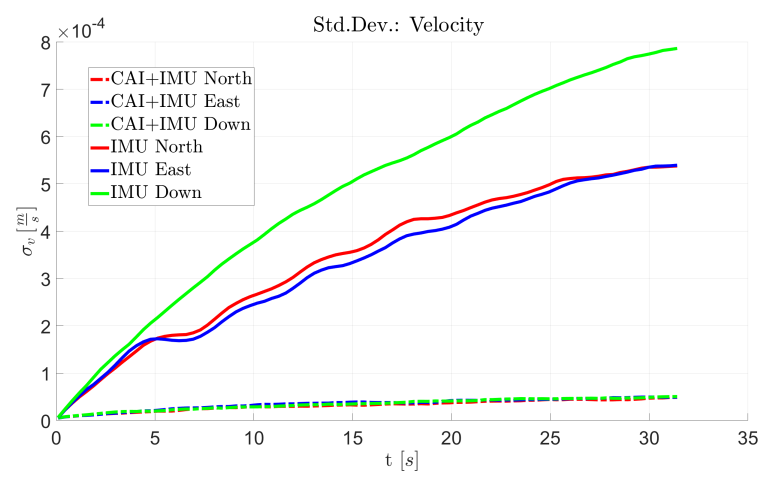

(d)

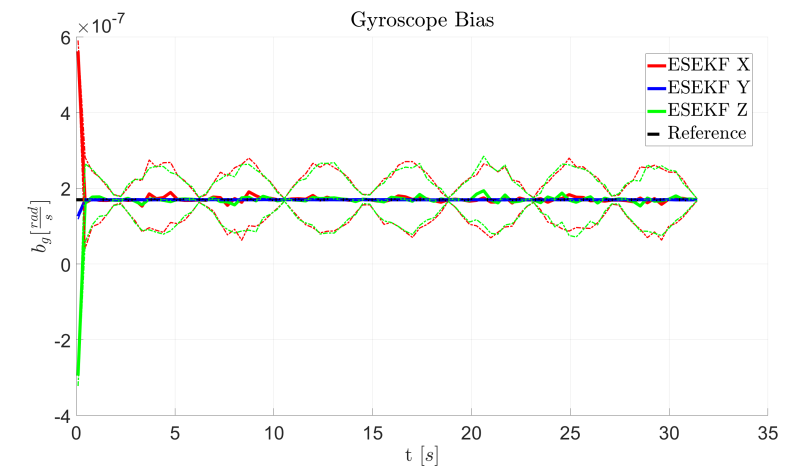

(c)

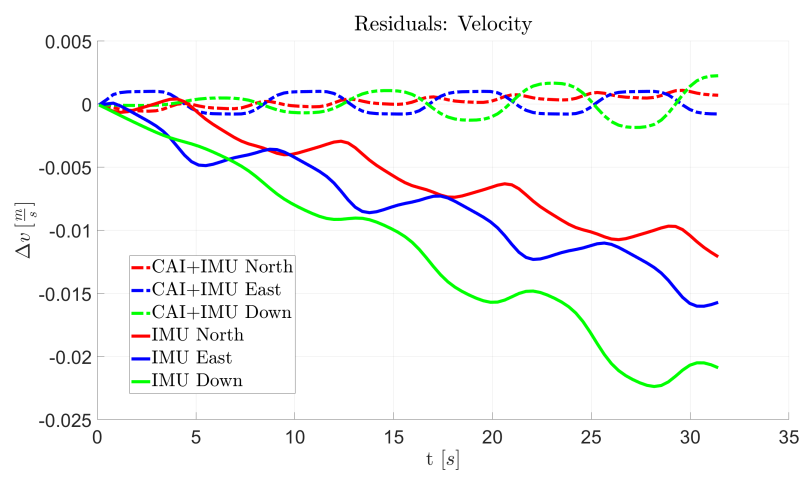

(e)

Fig. 6: Simulated slalom trajectory (a) with a velocity of $1 \mathrm{~m} / \mathrm{s}$ and major, sinusoidal changes of the azimutal orientation. The biases of accelereration (b) converges instantly. The gyroscope bias (c) also converges fast, but with larger uncertainties in the parts with the largest rate of change of the yaw angle, as indicated by the dotted lines. The uncertainty of the velocity state estimate is reduced by factor of 17 (d), and the systematic linear drift of the velocity reduced (e), while systematic effects caused by the averaging effect of the atom interferometer are visible. 
For a state to be observable, the corresponding column of the matrix needs to be different from zero. All entries of the matrix $\mathbf{H}$ are discussed now in order to determine the observability of the corresponding states.

a) Accelerometer and Gyroscope biases: Taking the example of the accelerometer bias, the derivative of the entries of $\mathbf{H}$ reads:

$$
\frac{\partial}{\partial \mathbf{b}_{\mathbf{a}}} A \cdot \cos (\boldsymbol{\Phi})=-\frac{1}{2} A T \mathbf{k} \cdot \sin (\boldsymbol{\Phi}) \frac{\partial \delta \mathbf{v}}{\partial \mathbf{b}_{\mathbf{a}}}
$$

This outer derivative of the cosine function is the same for every state. Since this term is coupled with the inner derivatives via chain rule, only the latter needs some further discussion.

The inner derivatives read:

$$
\begin{aligned}
\frac{\partial \delta \mathbf{v}}{\partial \mathbf{b}_{\mathbf{a}}} & =(\mathbf{I}-[\gamma \times]) T \\
\frac{\partial \delta \boldsymbol{\Psi}}{\partial \mathbf{b}_{\mathbf{g}}} & =(\mathbf{I}-[\gamma \times]) T
\end{aligned}
$$

It can be concluded that the accelerometer and gyro biases are always observable, unless $[\gamma \times]=\mathbf{I}$ which is impossible per definition. The remaining derivatives $\partial \delta \mathbf{v} / \partial \mathbf{b}_{\mathbf{g}}$ and $\partial \delta \boldsymbol{\psi} / \partial \mathbf{b}_{\mathbf{a}}$ are zero.

b) Misalignment: The partial derivative of the skewsymmetric matrix $[\gamma \times]$ w.r.t. its vector representation $\gamma=$ $\left[\gamma_{x}, \gamma_{y}, \gamma_{z}\right]^{T}$ in eq. (29)

$$
\begin{aligned}
\frac{\partial \delta \mathbf{v}}{\partial \boldsymbol{\gamma}} & =-\frac{\partial[\gamma \times]}{\partial \gamma}\left(\mathbf{f}^{b}+\mathbf{b}_{a}\right) T \\
\frac{\partial \delta \boldsymbol{\Psi}}{\partial \boldsymbol{\gamma}} & =-\frac{\partial[\gamma \times]}{\partial \boldsymbol{\gamma}}\left(\boldsymbol{\omega}^{b}+\mathbf{b}_{g}\right) T
\end{aligned}
$$

leads to another skew symmetric matrix eq. (30).

$$
\frac{\partial[\gamma \times]}{\partial \gamma}=\left(\begin{array}{ccc}
0 & 1 & -1 \\
-1 & 0 & 1 \\
1 & -1 & 0
\end{array}\right)
$$

This factor equals the negative pseudo vector of the term, hence eq. (29) equals to:

$$
\begin{array}{r}
\frac{\partial \delta \mathbf{v}}{\partial \boldsymbol{\gamma}}=\left[\mathbf{f}^{\prime b} \times\right] T \\
\frac{\partial \delta \boldsymbol{\Psi}}{\partial \boldsymbol{\gamma}}=\left[\boldsymbol{\omega}^{\prime b} \times\right] T
\end{array}
$$

with the forces and turn rates replaced by $\mathbf{f}^{\prime b}=\mathbf{f}^{b}+\mathbf{b}_{\mathbf{a}}$ and $\boldsymbol{\omega}^{\prime b}=\boldsymbol{\omega}^{b}+\mathbf{b}_{\mathrm{g}}$.

In order to observe the misalignment on a certain axis, the presence of a turn rate or acceleration on one of the remaining axes is necessary. However, if the inertial forces on the perpendicular directions have the same quantity, they compensate each other and the term becomes zero.

c) Leverarms: Under consideration of the remaining terms, eq. (19) reads:

$$
\delta \mathbf{v}=(\mathbf{I}-[\gamma \times])\left(\mathbf{f}^{\prime b}-\dot{\boldsymbol{\omega}}^{\prime b} \times \mathbf{l}-\boldsymbol{\omega}^{\prime b} \times \boldsymbol{\omega}^{\prime b} \times \mathbf{l}\right) T
$$

In line with the prior assumption of a time-invariant system during the interval of $T$, the Euler-term $\dot{\boldsymbol{\omega}}^{\prime b} \times \mathbf{l}$ is neglected for the derivative $\frac{\partial \delta \mathbf{v}}{\partial \mathbf{l}}$. This results in:

$$
\frac{\partial \delta \mathbf{v}}{\partial \mathbf{l}}=-(\mathbf{I}-[\gamma \times]) \frac{\partial}{\partial \mathbf{l}}\left(\boldsymbol{\omega}^{\prime b} \times \boldsymbol{\omega}^{\prime b} \times \mathbf{l}\right) T
$$

The interesting factor is the second one whose derivative can be proven to equal

$$
\frac{\partial \delta \mathbf{v}}{\partial \mathbf{l}}=\left[\boldsymbol{\omega}^{\prime b} \times\right]^{2} T
$$

The double cross product leads to a number of coupled terms on each axis. If the products $\omega_{x} \omega_{y} \approx \omega_{y} \omega_{z} \approx \omega_{x} \omega_{z} \approx 0$ are neglected, the expression $\left[\boldsymbol{\omega}^{\prime b} \times\right]^{2}$ can be interpreted as $\left[\boldsymbol{\omega}^{\prime b} \times\right]$ with its elements piece-wise squared. The keen reader may validate this for himself.

After this little thought experiment it can be stated that in order to observe the leverarm on one axis, a turn rate on either of the two remaining axes needs to be present. However, since each term is squared, small values like the Earth turn rate may not be sufficient to have any impact. On the other hand, values larger than 1 lead to a strong sensitivity.

The complete observation matrix with the misalignment factors omitted reads:

$$
\mathbf{H}^{T}=\left(\begin{array}{cc}
\mathbf{K}_{A} \mathbf{I} T & \mathbf{K}_{B} \mathbf{I} T \\
\mathbf{L}_{A} \mathbf{I} T & -\mathbf{L}_{B} \mathbf{I} T \\
\mathbf{K}_{A}\left[\mathbf{f}^{\prime b} \times\right] T+\mathbf{L}_{A}\left[\boldsymbol{\omega}^{\prime b} \times\right] T & \mathbf{K}_{B}\left[\mathbf{f}^{\prime b} \times\right] T-\mathbf{L}_{B}\left[\boldsymbol{\omega}^{\prime b} \times\right] T \\
\mathbf{K}_{A}\left[\boldsymbol{\omega}^{\prime b} \times\right]^{2} T & \mathbf{K}_{B}\left[\boldsymbol{\omega}^{\prime b} \times\right]^{2} T
\end{array}\right)
$$

with $\mathbf{K}_{i}=-\frac{1}{2} A T \mathbf{k} \cdot \sin \left(\boldsymbol{\Phi}_{i}\right), \mathbf{L}_{i}=-A T \mathbf{k} \cdot \overline{\boldsymbol{\nu}} \cdot \sin \left(\boldsymbol{\Phi}_{i}\right), i \in$ $\{A, B\}$

This also summarizes the requirements for the observability of the augmented states. For the leverarm estimation, a turn rate needs to be present on any perpendicular axis. As for the misalignment, either an acceleration or turn rate on a perpendicular axis is necessary.

It can be further stated that the system is stable as long as full observability is given, which might not be the case for all trajectories. However, the reduced state vector with only the kinematic states and the biases is safe to use in any application.

\section{CONCLUSION}

In this paper a promising hybrid filter solution based on the measurement of a cold atom interferometer as well as a conventional IMU was demonstrated by means of Monte Carlo simulation studies.

In contrast to most other filter attempts the spatial displacement of the atoms is calculated by a strapdown algorithm, which allows for a precise phase shift calculation in scenarios with a constant rate of change of the acceleration, rather than just constant values. Furthermore, this opens the opportunity to estimate the spatial dimensions of the interferometer chamber when developing such a sensor for certain dynamic conditions.

The simulations reveal a vast reduction of the drift of the filtered velocity state due to the corrected systematic errors of the conventional IMU. Also the precision of the solution 
expressed by the empirical standard deviation has improved as compared to the strapdown solution of the conventional IMU alone.

In scenarios with higher order change of accelerations, this deeper level system integration allows for a first order representation of the true trajectory. This still reduces the systematic error in comparison to the constant value assumption. On example of a slalom trajectory with a sinusoid signal of the yaw turn rate it was shown that the filter is able to accurately track the accelerometer bias, and even the gyroscope bias within systematic changing error bands, which was not possible before.

The observability of additional errors like misalignment and leverarms was discussed. Since certain dynamics are needed and furthermore it is hard to stabilize the filter with full state vector and only six CAI observations at a time, the ideal use case would be to determine the systematic displacement before the actual kinematic experiment, and then only use the bias states to account for any additional offsets. Another option includes the state augmentation with the CAI phaseshift rate of change like presented in [5]. This produces additional observations over to the system model and may improve the stability of the filter.

\section{REFERENCES}

[1] M. Kasevich, and S. Chu, "Atomic velocity selection using stimulated Raman transitions," Phys. Rev. Lett., 67, pp. 181-184, 1991.

[2] C. Jekeli, "Navigation error analysis of atom interferometer inertial sensor," Navigation, vol. 52, pp. 1-14, 2005.

[3] C. Freier et al.,"Mobile quantum gravity sensor with unprecedented stability," J. Phys.: Conf. Ser. 723 012050, 2016.

[4] Muquans (2019), "AQG Absolute Quantum Gravimeter", Datasheet, Muquans, Talence, Bordeaux, France, URL: https://www. muquans.com/wp-content/uploads/2019/ 03/muquans_aqg.pdf(visited 30.04.2021)

[5] P. Cheiney et al., "Navigation-compatible hybrid quantum accelerometer using a Kalman filter," arXiv:1805.06198v1, 2018.

[6] L. Richardson et al., "Opto-mechanical resonator-enhanced atom interferometry", February 2019, arXiv:1902.02867v1.

[7] B. Tennstedt and S. Schön, "Dedicated Calculation Strategy for Atom Interferometry Sensors in Inertial Navigation," 2020 IEEE/ION Position, Location and Navigation Symposium (PLANS), 2020, pp. 755-764, doi: 10.1109/PLANS46316.2020.9110142.

[8] A. Rakholia et al., "Dual-Axis High-Data-Rate Atom Interferometer via Cold Ensemble Exchange," Physical Review A, 2(5), 2014.

[9] P. G. Savage, "Strapdown Inertial Navigation Integration Algorithm Design Part 1: Attitude Algorithms," Journal of Guidance, Control and Dynamics, Vol. 21, No.1 Jan-Feb 1998.

[10] P. G. Savage, "Strapdown Inertial Navigation Integration Algorithm Design Part 2: Velocity and Position Algorithms," Journal of Guidance, Control and Dynamics, Vol. 21, No.2 Mar-Apr 1998.

[11] A. Gauguet et al., "Characterization and limits of a cold atom Sagnac interferometer," Phys. Rev. A 80, 063604, December 2009.

[12] G. Tackmann et al., "Large-area Sagnac atom interferometer with robust phase read out," Comptes Rendus Physique Vol. 15, Issue 10, pp. 884897, 2014.

[13] Ch. Antoine and Ch. J. Bordé 2003 J. Opt. B: Quantum Semiclass. Opt. $5 \mathrm{~S} 199$

[14] D. Titterton, J. Weston, "Strapdown Inertial Navigation Technology," Institution of Engineering and Technology, 2004.

[15] B. Barrett et al., "Mobile and remote inertial sensing with atom interferometers," International School of Physics "Enrico Fermi" on Atom Interferometry, Vol. 188, Varenna, Italy, 2014.

[16] P. Berg, "Ein rauscharmes Materiewellen-Gyroskop hoher Genauigkeit,"phd thesis, Leibniz Universität Hannover, 2014.
[17] Gersemann, M., Gebbe, M., Abend, S. et al. Differential interferometry using a Bose-Einstein condensate. Eur. Phys. J. D 74, 203 (2020). https://doi.org/10.1140/epjd/e2020-10417-8.

[18] B. Canuel et al., "Six-axis inertial sensor using cold-atom interferometry," Phys. Rev. Lett., 97, 010402:1-010402:4, 2006.

[19] X. Wang et al., "Enhancing Inertial Navigation Performance via Fusion of Classical and Quantum Accelerometers", March 2021, arXiv:2103.09378.

[20] iMAR (2021), "iNAT-RQT-4001", Datasheet, IMAR Navigation and Control, St. Ingbert, Saarland, Germany, URL: https://imar-navigation.de/downloads/NAT_RQT_400 x_en.pdf(visited 30.04.2021)

[21] B. Tennstedt, C. Schubert, D. Schlippert, S. Schön and E. M. Rasel, "Impact of Uncertainties in Atom Interferometry on Strapdown Navigation Solutions," 2019 DGON Inertial Sensors and Systems (ISS), 2019. pp. 1-20, doi: 10.1109/ISS46986.2019.8943632. 